\title{
METHODS OF ATTOSECOND X-RAY PULSE GENERATION
}

\author{
Alexander Zholents, LBNL, Berkeley, CA94720, U.S.A.
}

\section{Abstract}

We review several proposals for generation of solitary attosecond pulses using two types of free electron lasers which are envisioned as future light sources for studies of ultra-fast dynamics using soft and hard x-rays.

\section{INTRODUCTION}

Attitudes toward attosecond $\mathrm{x}$-ray pulse production have changed dramatically over the past several years. Not long ago x-ray pulses with a duration of a few hundred attoseconds were just science fiction, but today they are already a tool for some researchers. Breakthrough progress in the generation of solitary extreme ultraviolet pulses of several hundred attosecond duration has been made by the laser community [1-5]. Following this lead, people in the free electron laser community have begun to develop new ideas on how to generate attosecond x-ray pulses in the soft and hard x-ray energy ranges.

Two types of free electron lasers (FELs) are considered for production of soft and hard X-rays: (i) a high-gain harmonic generation (HGHG) FEL (see, [6,7] and references therein), and its companion low-gain harmonic cascade (HC) FEL [8-10], and (ii) a high-gain self amplified spontaneous emission (SASE) FEL (see, a review paper [11] and references therein). In the following discussion we assume some knowledge of these FELs and in particular concepts of FEL resonance, slippage and FEL gain. Useful information can be found in [12].

Schemes for generation of attosecond x-ray pulses have been recently proposed for both types of the FELs. In most cases they can be realized with a little modification to the main facility. Except for the first proposal (defined below) all other proposals employ interaction of electrons with a few-cycle laser pulse with carrier-envelope phase stabilization in a short wiggler magnet consisting of just one or two periods. This interaction plays a key role for eventual generation of the attosecond $\mathrm{x}$-ray pulses with free electron lasers. It also links the attosecond x-ray pulse to the laser pulse, thus providing an opportunity for accurate synchronization between the laser pump pulse and $\mathrm{x}$-ray probe pulse for pump-probe experiments.

\section{FIRST PROPOSAL}

A technique that uses elements of SASE FEL and HGHG FEL was proposed in [13]. For future reference we will call this Proposal A. The idea is to exploit the spiky temporal structure of the SASE output where the width of individual spikes is of the order of few hundred attoseconds. It was noted that after harmonic multiplication to the eighth harmonic, the original SASE intensity fluctuations are transformed to a situation where often a single spike nearly completely dominates the entire radiation output. Calculations predict a probability of $1-10 \%$ for an approximate contrast ratio of $1: 1$ (defined as the ratio of the energy of the dominant spike to the energy of the rest of the x-ray pulse). Selection of such events can be achieved by analyzing a record of the radiation intensity on a pulse-to-pulse basis. Good contrast corresponds to an $\mathrm{x}$-ray pulse intensity which is more than twice as large as the average intensity taken over many pulses. Because of the random nature of the SASE spikes, the dominant spike may appear temporally anywhere inside of the electron bunch. This complicates synchronization of the attosecond pulse to any external pulse in a pump-probe experiment.

The evident inconvenience of the described method made it obsolete as new ideas for attosecond x-ray pulses generation appeared. These ideas have much in common between them and with earlier experiments in the generation of the attosecond pulses at extreme ultraviolet wavelengths; namely, all of them use a few-cycle optical pulse with a carrier-envelope phase stabilization.

\section{METHOD BASED ON HC FEL}

A unique opportunity that a few-cycle optical pulse has to offer for generation of attosecond x-ray pulses using free electron lasers was first recognized in [14]. A technique described there and in an expanded form in [15] requires an ultra-relativistic electron beam, a few-cycle, intense optical laser pulse and an intense pulse of coherent x-ray radiation, together with a number of magnetic undulators and transport elements. We call this Proposal B. Fig.1 schematically shows how all these components are used. On the left is a source producing a coherent 2-nm wavelength, 100-fs, 100-MW peak power x-ray pulses. While such sources do not exist today, studies of HC FEL's [8-10] have suggested approaches which are feasible in principle. As a specific example the x-ray source at 2-nm wavelength is chosen to eventually produce 1-nm wavelength attosecond radiation. However, attosecond pulse generation at both longer and shorter wavelengths is also possible with the same technique.

The HC FEL can be configured such that only part of the electron bunch is used for the X-ray generation, thus leaving another part near the bunch head whose instantaneous energy spread $\sigma_{E}$ has not been degraded by previous FEL interaction in the upstream cascade.

After exiting the HC FEL, an achromatic bend inserts the electrons into a two-period wiggler magnet " $800-\mathrm{nm}$ modulator". Simultaneously, a 800-nm wavelength, 1 $\mathrm{mJ}, 5$-fs laser pulse enters this wiggler and co-propagates with the electrons. The technical feasibility of such optical pulses has already been proven [16]. The relative timing between the arrival of the electron beam and the optical pulse is set such that the latter temporally overlaps "virgin" electrons. We presume that the x-ray HC FEL pulse will be seeded with a laser pulse which originates 


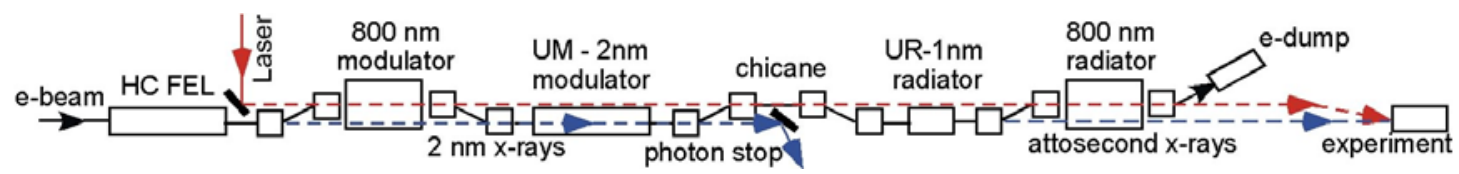

Figure 1. A schematic of the components involved in attosecond x-ray pulse production using the HC FEL.

from the same laser source as the few-cycle laser pulse which consequently permits tight synchronization between the two. Since the "virgin" ultra-relativistic electrons and the HC FEL x-ray pulse come from the same electron bunch, one can thus ensure temporal synchronization between each of these three beams.

The carrier-envelope phase of the few-cycle laser pulse is adjusted so that the peak electric field appears at the peak of the envelope when the laser pulse passes the wiggler center. The wiggler's magnetic period and undulator parameter $K$ (see, [12]) are adjusted such that fundamental FEL resonance occurs at the laser wavelength $\lambda_{L}=800 \mathrm{~nm}$ at electron beam energy of 3 $\mathrm{GeV}$. The interaction with the laser light in the wiggler then produces a time-dependent electron energy modulation extended over few optical cycles shown in Fig.2. For the laser pulse parameters mentioned above, we expect a central peak energy offset $\Delta \mathrm{E}_{0}=15 \mathrm{MeV}$ which is a factor of 1.35 times larger than those of its two nearest neighbors. This relative difference is important when considering the 2-nm energy modulation to be induced in a following undulator.

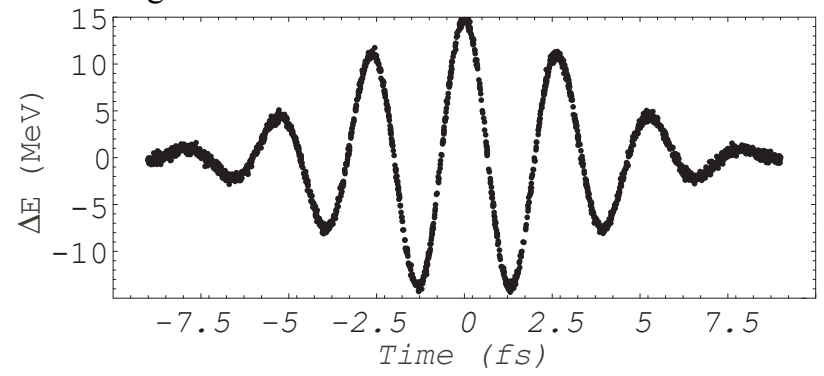

Figure 2. The calculated energy modulation of the electrons along the electron bunch produced in the interaction with a few-cycle, $800 \mathrm{~nm}$ laser pulse.

A second isochronous bend after the wiggler magnet returns the electrons back to the original axis. The electrons now enter a long undulator-modulator (UM), which serves as an energy modulator at 2-nm wavelength. The coherent, $\geq 100$-fs long, 2 -nm output pulse from the HC FEL co-propagates in the UM with electrons and arrives simultaneously with those electrons that experienced the strong energy modulation at $800 \mathrm{~nm}$. The undulator parameter $K$ of the UM is tuned such that only those electrons very near the peak of the 800-nm energy modulation have the correct energy for resonant FEL interaction with the 2-nm light. The other electrons fall outside the energy bandwidth of the UM and are not significantly modulated.

Downstream of the UM the electrons enter a chicane with a time-of-flight parameter $R_{56}=750 \mathrm{~nm}$ which induces strong microbunching at $\lambda_{x}=2-n m \quad x$-ray wavelength and at higher harmonics $\lambda_{x} / \mathrm{n}$. Skipping details of the mathematical analysis that can be found in [14] we plot in Fig. 3 the bunching amplitude for $\mathrm{n}=2$, i.e. $1-\mathrm{nm}$ wavelength, as theoretically predicted and as calculated by the GINGER [17] simulation code.

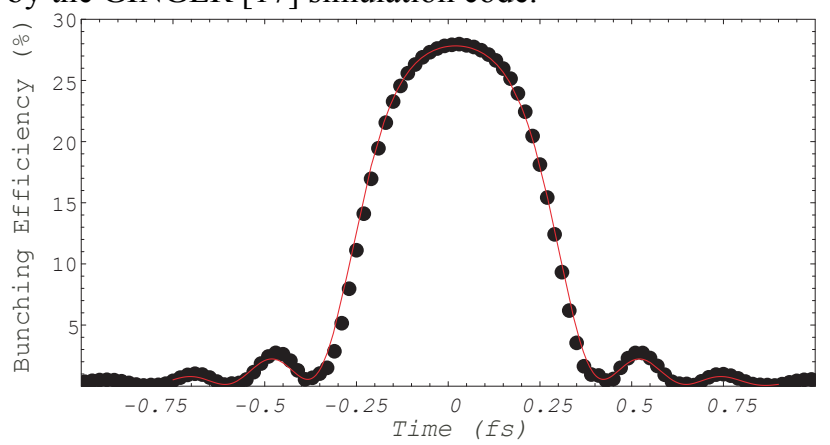

Figure 3. Bunching efficiency at $1 \mathrm{~nm}$ versus time along the electron bunch. The solid line shows the analytical prediction, and the dots show simulation results from Ginger. The FWHM of the peak is 530 attoseconds.

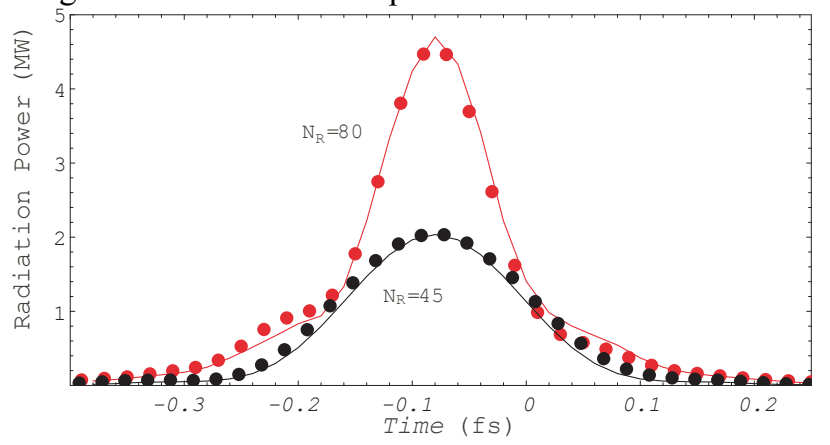

Figure 4. Predicted attosecond pulse power at $1 \mathrm{~nm}$ from a radiator with 80 periods (top line) and 45 periods (bottom line). Both curves were normalized to the peak intensity of 80 periods simulation results (dots).

After the chicane, the electrons proceed to an undulatorradiator (UR) in micro-bunches and produce coherent emission at wavelength $\lambda_{x}=1 \mathrm{~nm}$. The interference of the waves emitted by all macroparticles defines the output envelope of the radiation field. The predicted radiation field intensity is shown in Fig.4. The rms width $\sigma_{t}$ of the peak is 48-attosecond (asec) for the radiator with number of periods $N_{R}=80$ and 75 -asec for the radiator with $N_{R}$ $=45$. This is several times shorter than the bunching width structure shown in Fig.3. This reduction is attributed to a destructive interference (due to temporal variation of bunching phase) occurring between waves emitted by microbunches on opposite sides of the bunching peak.

The described method and two following methods described in the next paragraph share a certain similarity with "slicing" approach [18] currently used for selection 
of the femtosecond x-ray pulses of spontaneous electron emission. For this reason we call them slicing methods.

\section{METHODS BASED ON SASE FEL}

\section{Slicing Methods}

A technique for generation of attosecond $\mathrm{x}$-ray pulses has been also proposed for SASE FELs [19, 20]. It resembles the above described scheme of Proposal B, but a "800-nm modulator" is now placed before an x-ray undulator used for SASE. A few-cycle laser pulse with a stabilized carrier-envelope phase co-propagates through the undulator with the electron bunch and produces an energy modulation with a peak amplitude $\Delta \mathrm{E}_{0} \sim 30-40$ $\mathrm{MeV}$. This modulation is more than two times larger than in the previous technique and consequently requires more than four times laser pulse energy. Although, to the best of our knowledge, lasers producing such pulses do not exist today, it is reasonable to expect them to be developed in the near future considering rapid progress in ultra-fast laser technology.

After the "800-nm modulator" the electron bunch proceeds into the long $x$-ray undulator where the SASE process develops. Electrons located at the peak of the energy modulation amplitude emit at the offset frequency $\omega+\delta \omega$, where $\delta \omega / \omega=\Delta \mathrm{E}_{0} / \mathrm{E}=5 \times 10^{-3}$, and unmodulated electrons from the rest of the bunch emit x-rays at a reference frequency $\omega$. This frequency separation allows a selection of a single attosecond pulse produced by the electrons at the peak of energy modulation using a monochromator [19]. For a future reference, we call this technique Proposal C.

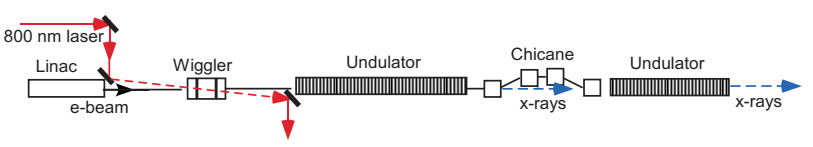

Figure 5. A schematic of the components involved in attosecond $\mathrm{x}$-ray pulse production using SASE FEL and a selection technique based on energy modulation of electrons.

As a further extension of Proposal C, a more elaborate approach for a selection of the attosecond pulse out of the output signal of the FEL that works without monochromator was proposed in [20]. A schematic of a new technique which we call as the Proposal D is shown in Fig.5. The idea is to divide the x-ray undulator into two uneven parts with the first part being a source for a seed signal to be used in the second part. A time delay magnetic chicane similar to the time delay chicane between adjacent stages in the HC FEL allows for a seed signal to interact with "virgin" electrons from the same electron bunch in the second undulator. The second undulator is tuned such as to favor the SASE process at the frequency corresponding to the offset frequency of the radiation produced in the first undulator by the electrons at the peak of the energy modulation amplitude. This is a key element for a selection of the single attosecond pulse since then the seed radiation at the offset frequency is exponentially amplified, but seed radiation at the reference frequency is not. An example of the predicted attosecond x-ray pulse is shown in Fig. 6. Calculations using the FEL code FAST [21] show a FWHM pulse duration of $\sim 300$ asec, a significant output peak power of the order of $10 \mathrm{GW}$ (which can be increased up to 100 GW using a tapered undulator), and an approximate ratio of the attosecond peak power to background power of 400:1 [20] corresponding to a contrast of the attosecond pulse of 1.3:1 for a 100-fs long electron bunch.

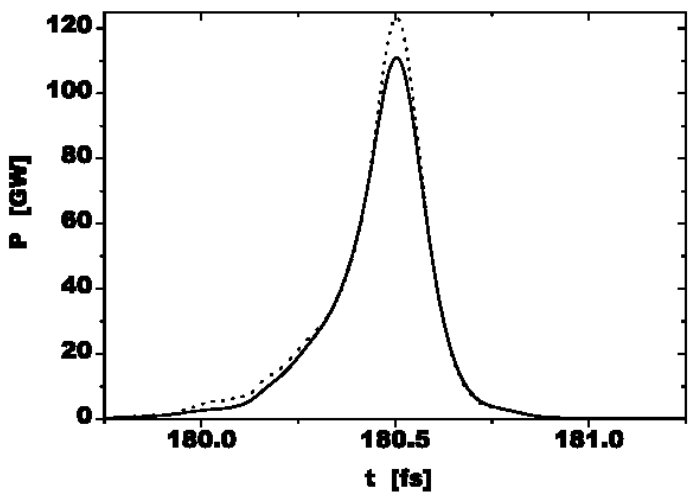

Figure 6. A predicted attosecond pulse for the slicing scheme shown in Fig.5. Case with monocromator (solid line) and case without monochromator (dashed line).

\section{Current Enhancement Method}

Another technique for a selection of single attosecond $\mathrm{x}$-ray pulses in the SASE FEL has been proposed in [22]. Fig.7 shows a schematic of this technique which we call Proposal E. On the left, the electron beam exits the linac

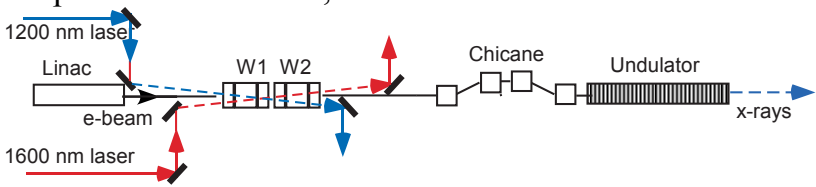

Figure 7. A schematic of the components involved in attosecond x-ray pulse production using SASE FEL and a selection technique based on the electron peak current enhancement.

and enters two adjacent wiggler magnets, labeled $\mathrm{W} 1$ and W2, where each magnet has just one wiggler period. Two co-propagating laser pulses with $1200-\mathrm{nm}$ wavelength ( $0.2 \mathrm{~mJ}$ pulse energy and $7.5 \mathrm{fs}$ FWHM) and $1600-\mathrm{nm}$ wavelength $(0.07 \mathrm{~mJ}$ and $10 \mathrm{fs})$ enter the wigglers at a small angle. The above laser parameters have not yet been demonstrated experimentally. The first laser pulse is focused in the center of the first wiggler and the second laser pulse is focused in the center of the second wiggler. Each laser interacts with the same group of electrons at its focal point. For the first laser pulse, the phase of the carrier wave is adjusted so that the electric field is zero when the peak of the laser pulse envelope reaches the center of the first wiggler. The phase of the carrier wave of the second laser pulse is adjusted so that the electric field is zero when the peak of the laser pulse envelope 
reaches the center of the second wiggler. A technique for obtaining such pulses using an Optical Parametric Amplifier is described elsewhere [23-26].

The interaction of the $14 \mathrm{GeV}$ electron beam with the two laser fields in the two wigglers results in a complex time-dependent energy modulation of the electrons shown in Fig.8.

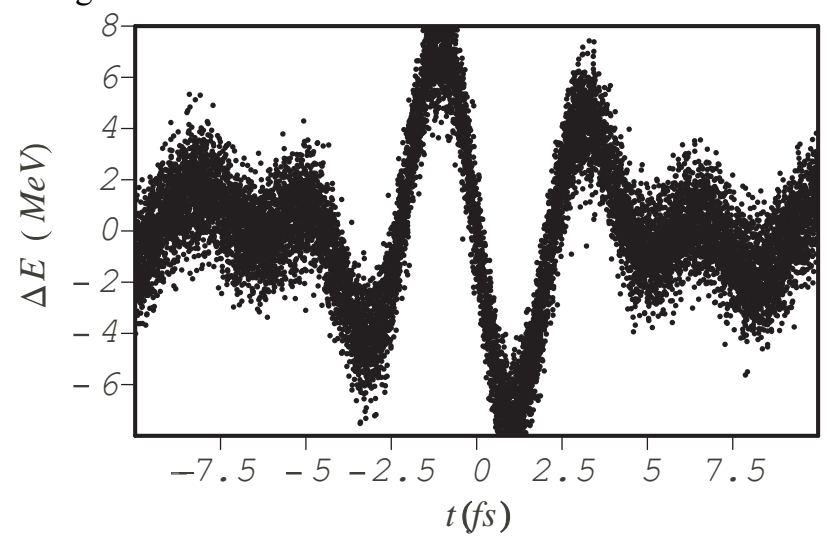

Figure 8. A combined energy modulation produced in interation with a few-cycle $1200 \mathrm{~nm}$ laser pulse in one wiggler magnet and a few-cycle $1600 \mathrm{~nm}$ laser pulse in a second wiggler magnet.

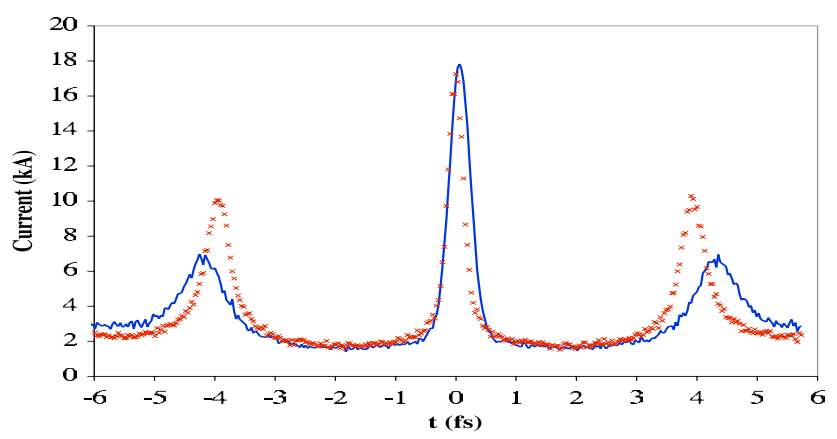

Figure 9 Current profile resulting from intereaction with two lasers (blue line) and with a single laser (red crosses).

After modulation, the electron beam passes through a dispersive magnetic chicane that produces a microbunching of the electrons and enhancement of the electron peak current as shown in Fig.9. The current enhancement produced in the central cycle of the two laser pulses is almost two times stronger than the current enhancement produced during other laser cycles. For comparison, the current enhancement produced after modulation with a single 1200-nm laser is also shown.

The radiation produced by the modulated, bunched electron beam has been simulated using the FEL code GENESIS [27] using the same electron beam and undulator parameters as in the design report for Linac Coherent Light Source [28], except the beam is focused using a FODO lattice having an average beta function of $18 \mathrm{~m}$. The SASE process for the main and secondary current peaks was simulated up to 20 times using different random seeds for the shot noise within the electron beam. The statistics for the power profile of the main pulse are summarized in Fig.10. The large fluctuations are a result of variations in both the total energy per pulse and changes in the temporal pulse shape. The contrast ratio in terms of x-ray energy of the main current peak compared with the energy from the side peaks and from 100-fs of

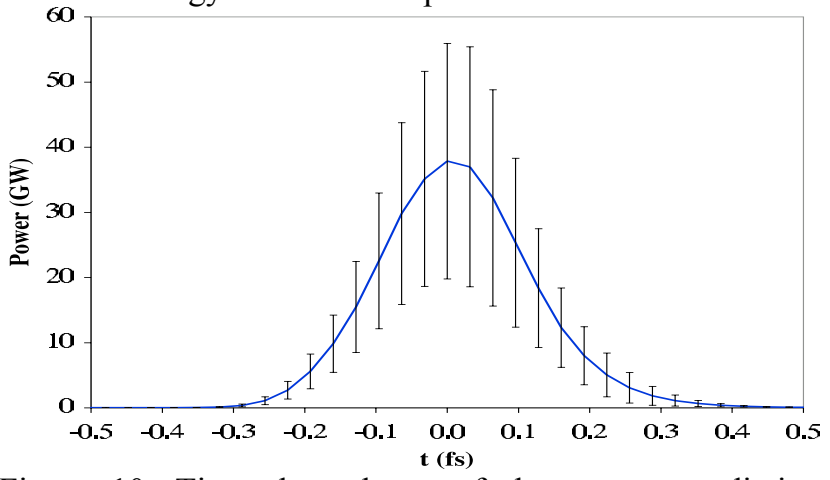

Figure 10. Time dependence of the average radiation power with statistical deviations arising from different random seeds for the shot noise.

unperturbed beam is shown as a function of distance along the undulator in Fig.11. After $50 \mathrm{~m}$ of undulator, the background energy from the bulk of the beam is about 6 $\mu \mathrm{J}$. The main peak averages $9.8 \mu \mathrm{J}$ of energy while each side peak averages about $1 \mu \mathrm{J}$ of energy. Each x-ray pulse exhibits roughly $40 \%$ variation between simulations which use different random seeds for the shot noise. The total contrast ratio between the main current peak and the combined sources of radiation from the rest of the beam is approximately $1: 1$.

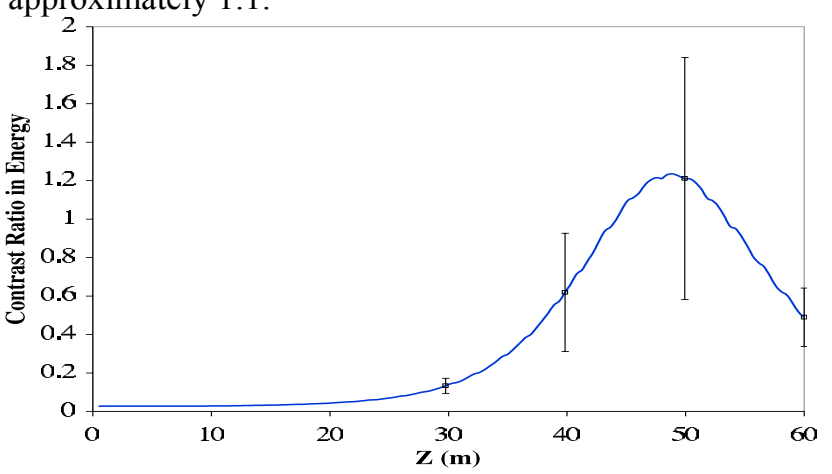

Figure 11. Contrast ratio in terms of $\mathrm{x}$-ray energy between the main current peak and the rest of the electron beam, including side peaks. Statistical fluctuations are indicated for several locations in $\mathrm{z}$.

\section{CONCLUSION}

In Table 1 we outline distinct features for each of the five proposals for generation of attosecond pulses using FELs discussed here. Among them, we favor Proposals B, D, and E. Proposal A lacks the ability of synchronization to the laser and has a low $1-10 \%$ yield of shots with attosecond pulses. Proposal $\mathrm{C}$ was superseded by the Proposal D which practically works the same way, but does not rely upon a monocromator for a selection of the attosecond pulses (although it can use a monochromator to improve contrast of the attosecond pulse). All three proposals B, D, and $\mathrm{E}$ use a laser pulse to initiate 
generation of attosecond $\mathrm{x}$-ray pulses. This leads to an absolute synchronization between the attosecond x-ray pulse and laser pulse, which is essential for experiments with attosecond $\mathrm{x}$-ray pulses.

Overall, it is now evident that both soft and hard x-ray pulses with duration of the order of $(200-300)$ asec in the case of the SASE FEL and of the order of (100-150) asec in the case of the HC FEL can be produced. Proposals D and E for SASE FEL's require more energy in few-cycle pulses than presently demonstrated, i.e. 3-4 $\mathrm{mJ}$ at $800 \mathrm{~nm}$ wavelength in the case of the Proposal D and $0.2 \mathrm{~mJ}$ at $1200 \mathrm{~nm}$ wavelength in the case of the Proposal E. The capability of OPA`s to produce few-cycle pulses also requires an experimental proof.

Table 1. Comparison of five techniques for generation of attosecond pulses.

\begin{tabular}{|l|c|c|c|c|c|}
\hline Proposal & $\begin{array}{c}\text { A } \\
\text { Ref.[13] }\end{array}$ & $\begin{array}{c}\text { B } \\
\text { Ref.[14] }\end{array}$ & $\begin{array}{c}\text { C } \\
\text { Ref. [19] }\end{array}$ & $\begin{array}{c}\text { D } \\
\text { Ref.[20] }\end{array}$ & $\begin{array}{c}\text { E } \\
\text { Ref.[22] }\end{array}$ \\
\hline FEL type & SASE+HC & HC & SASE & SASE & SASE \\
\hline $\begin{array}{l}\text { Numerical example } \\
\text { shown for a wavelength }\end{array}$ & $0.15 \mathrm{~nm}$ & $1 \mathrm{~nm}$ & $0.15 \mathrm{~nm}$ & $0.15 \mathrm{~nm}$ & $0.15 \mathrm{~nm}$ \\
\hline $\begin{array}{l}\text { Absolute synchronization to } \\
\text { external optical laser }\end{array}$ & No & Yes & Yes & Yes & Yes \\
\hline Needs optical laser development & N/A & No & Yes & Yes & Yes \\
\hline$\%$ of useful shots & $1-10$ & 100 & 100 & 100 & 100 \\
\hline Needs monochromator & No & No & Yes & No & No \\
\hline FWHM pulse duration, asec & 300 & $100-150$ & 300 & 300 & 250 \\
\hline Peak power & $10 \mathrm{GW}$ & $10 \mathrm{MW}$ & $10 \mathrm{GW}$ & $10-100 \mathrm{GW}$ & $40 \mathrm{GW}$ \\
\hline Contrast of attosecond pulse & $\sim 1$ & $>>1$ & $\sim 1$ & $\sim 1$ & $\sim 1$ \\
\hline
\end{tabular}

The attosecond pulses that can be produced with the SASE FEL have a huge peak power of the order of 10-40 GW which can be increased up to $100 \mathrm{GW}$ if a tapered undulator is used. The HC FELs are typically less powerful devices and attosecond pulses that can be produced with the HC FEL are of the order of $10 \mathrm{MW}$ (at ten times smaller photon energy and approximately five times smaller peak electron current).

\section{ACKNOWLEDGEMENTS}

We are grateful to W. Fawley and G. Penn with whom many of ideas discussed here were developed together. This work was supported by the Director, Office of Science, of the U.S. Department of Energy under Contract No. DE-AC03-76SF00098.

\section{REFERENCES}

[1] T. Brabec and F. Krausz , Rev. Mod. Phys., 72, 545 (2000).

[2] M.Hentchel et al., Nature, 416, 509 (2001).

[3] M.Drescher et al., Nature, 419, 803 (2002).

[4] A. Baltuška et al., Nature, 421, 611 (2003).

[5] R.Kienberger et al., Nature, 427, 817 (2004).

[6] R. Bonifacio, L. De Salvo Souza, E.T. Scharlemann, NIM A, 296, 787(1990).

[7] L.-H. Yu et al., Science, 289, 932 (2000).

[8] W. Fawley et al., 2003 Part. Acc. Conf., IEEE 03CH37423, 923 (2003).

[9] G. Penn et al., in Proc. of European Part. Acc. Conf. , Luzerne (2004), available at http://www.JACoW.org.
[10] G. Penn, LBNL-44973, (2004).

[11] C. Pellegrini, NIM A, 475, 1 (2001).

[12] W. Colson, C. Pellegrini, A.Renieri Ed's, Laser Handbook, V.6, North Holand, (1990).

[13] E.L. Saldin, E.A. Schneidmiller, and M.V. Yurkov, Opt. Comm., 212, 377 (2002).

[14] A.A. Zholents, W.M. Fawley, Phys. Rev. Lett., 92, 224801 (2004).

[15] A.A. Zholents, W. M. Fawley, Proc. of SPIE, 5534, 108 (2004).

[16] L S. Sartania et al., Opt. Lett., 22, 1562 (1997).

[17] W.M. Fawley, LBNL Tech. Rpt. 49625, (2001).

[18] S. Khan, this conference, contribution TOAB007.

[19] E.L.Saldin, E.A.Schneidmiller, and M.V.Yurkov, Opt. Comm., 237, 153 (2004).

[20] E.L.Saldin, E.A.Schneidmiller, and M.V.Yurkov, Opt. Comm., 239, 161 (2004).

[21] E.L.Saldin, E.A.Schneidmiller, and M.V.Yurkov, NIM A., 429, 233 (1999).

[22] A.A. Zholents, G. Penn, LBNL-56773, (2005), submitted to Phys. Rev. ST $-A B$.

[23] T. Kobayashi and K. Torizuka, Opt. Lett., 26, 1295 (2001).

[24] A. Baltuška, et al., Opt. Lett., 27, 1241 (2002).

[25] X. Fang and T. Kobayashi, Opt. Lett., 29, 1282 (2004).

[26] C.P. Hauri et al., Opt. Lett., 29, 1369 (2004).

[27] S. Reiche, NIM A, 429, 243 (1999).

[28] See, e.g., "Linac Coherent Light Source Design Study Report", SLAC-R-521, 1998. 
